\title{
Study of surfaces and interfaces using quantum chemistry techniques
}

\author{
William A. Goddard III and T. C. McGill \\ Arthur Amos Noyes Laboratory of Chemical Physics ${ }^{a}$ and Harry G. Steele Laboratory of Electrical Sciences, \\ California Institute of Technology, Pasadena, California 91125 \\ (Received 23 April 1979; accepted 29 May 1979)
}

There are a number of difficulties in elucidating the microscopic details of the electronic states at surfaces and interfaces. The first step should be to determine the structure at the surface or interface, but this is difficult experimentally even for the clean, ordered surface and extremely difficult for cases with impurity atoms (e.g., nonordered oxide layers). The theoretical study of such geometries and energy surfaces is the subject of quantum chemistry. We present a review of some of the theoretical techniques from quantum chemistry that are being applied to surfaces. The procedure consists of treating a finite piece of the surface or interface as a molecule. $A b$ initio calculations are then carried out on the molecule using the generalized valence bond (GVB) method (with additional configuration interaction), thereby incorporating the dominant many-body effects. The reliability of these techniques is discussed by giving some examples from molecular chemistry and the surfaces of solids. The strengths and weaknesses of this approach are compared with more traditional band theory related methods and are illustrated with various examples.

PACS numbers: 73.20. $-\mathrm{r}, 71.10 .+\mathrm{x}, 68.20 .+\mathrm{t}$

\section{INTRODUCTION}

A general goal of the surface scientist is to develop a microscopic description of the chemical processes, structure, and electronic states at surfaces and interfaces. In this endeavor theory promises to play a key role since the experiments are frequently difficult to interpret unambiguously and theory is required to allow comparison between various experimental results. Further, many important phenomena on surfaces involve short-lived chemical intermediates or reactions at defects or other centers present in small concentrations, making experimental detection very difficult.

To be maximally useful in elucidating important surface phenomena, the theory must be able to provide reliable answers concerning such questions as (i) the structure of surfaces, (ii) the structure, site, and energy for chemisorbed species and reaction intermediates (including cases with broken bonds), and (iii) the potential energy surfaces for the motion of the relevant atoms or molecules at the surface or interface. To provide a means of experimental verification of the theoretical results, it is important for the theory to characterize those properties of the surface or interface that might be observable experimentally. Thus the theory should predict atomic spacings, vibrational frequencies, photoemission spectra, and electronic excitation energies for the various species on the surface. These requirements place quite severe constraints upon the theory. Indeed, no theoretical methods currently satisfy all requirements; however, current quantum chemical techniques meet some of the important objectives and show promise of developments to satisfy the others.

In this paper we review some of the methods that have been applied in quantum chemistry to answer questions about chemical processes and structure. In Sec. II, we review some of the theoretical methods. Section III illustrates some problems and successes of these methods while Sec. IV contains a summary.

\section{THEORETICAL METHODS}

Over the last two decades, methods have been developed for accurate first principles calculations of the wavefunctions of molecules. ${ }^{1-7}$ These methods of quantum chemistry involve (i) exact evaluation of all one- and two-electron integrals involved in evaluating the energies of wavefunctions, and (ii) explicit consideration of all electrons (core and valence). Quantum chemical methods lead directly to total energies and hence can be used to obtain accurate geometries for ground states and excited states. Indeed, these methods can be used to obtain complete potential surfaces (including activation barriers) for reactions of molecules. In addition, these methods lead to direct evaluation of excitation energies, ionization potentials, and electron affinities.

Since the quantum chemical methods deal with explicit evaluation of the total energy of explicit wavefunctions, they are amenable to direct, sequential inclusion of electron correlation or many-body effects. This allows one to learn how sensitive various properties are to electron correlation and then to use the appropriate methods in various applications.

\section{A. Hartree-Fock \\ 1. Basic equations}

The most common approach to wavefunctions is the Hartree-Fock (HF) or molecular orbital method. For a simple molecule (referred to as closed-shell), this method involves placing two electrons (one of each spin) in a Slater determi- 
nant of molecular orbitals and optimizing the shape of the molecular orbitals to achieve the lowest energy. Thus the wavefunction for an $N$ electron (closed-shell) wavefunction is

$$
\left.\Psi_{\left(r, r_{2}, \ldots, r N\right)}^{\mathrm{CS}}=a\left[\phi_{1} \alpha\right)\left(\phi_{1} \beta\right)\left(\phi_{2} \alpha\right)\left(\phi_{2} \beta\right) \ldots\right],
$$

where $a$ is the antisymmetrizer or determinant operator and the $N / 2$ spatial orbitals are taken as orthonormal,

$$
\left\langle\phi_{\mathrm{i}} \mid \phi_{\mathrm{j}}\right\rangle=\delta_{\mathrm{ij}}
$$

The total energy of this wavefunction has the form

$$
E=2 \sum_{i=1}^{N / 2}\left(h_{i i}+J_{i i}\right)+\sum_{i>j=1}^{N / 2} 2\left(2 J_{i j}-K_{i j}\right)+\sum_{A>B} \frac{Z_{A} Z_{B}}{r_{A B}},
$$

where lower case letters refer to spatial orbitals and upper case letters refer to nuclei, where $h_{i i}=\left\langle\phi_{i}|h| \phi_{i}\right\rangle$ is the oneelectron energy of orbital $\phi_{i}$,

$$
h(1)=-1 / 2 \nabla_{1}^{2}-\sum_{A} Z_{A} / r_{A 1}
$$

is the part of the Hamiltonian pertaining to a particular electron $^{8}$ ( 1 in this case), where

$$
J_{i j}=\int d^{3} r_{1} \phi_{i}^{*}(1) \phi_{i}(1) \int d^{3} r_{2} \frac{\phi_{j}^{*}(2) \phi_{j}(2)}{r_{12}}
$$

is the Coulomb interaction between charge densities $\left|\phi_{\mathrm{i}}\right|^{2}$ and $\left|\phi_{\mathrm{j}}\right|^{2}$, and where

$$
K_{i j}=\int d^{3} r_{1} \phi_{i}^{*}(1) \phi_{j}(1) \int d^{3} r_{2} \frac{\phi_{j}^{*}(2) \phi_{i}(2)}{r_{12}}
$$

is the exchange interaction.

Noting that

$$
J_{i i}=K_{i i}
$$

Eq. (2) can be rewritten as

$$
E=E_{0}+E_{1}+E_{2},
$$

where

$$
\begin{gathered}
E_{0}=\sum_{A>B} \frac{Z_{A} Z_{B}}{r_{A B}}, \\
E_{1}=2 \sum_{i=1}^{N / 2} h_{i i},
\end{gathered}
$$

and

$$
E_{2}=2 \sum_{i, j=1}^{N / 2}\left(2 J_{i j}-K_{i j}\right)
$$

are the zero-electron, one-electron, and two-electron interactions.

Applying the variational principle to (7) leads to the necessary condition (the Euler-Lagrange equation) for the optimum orbitals,

$$
H^{\mathrm{HF}} \phi_{i}=\epsilon_{i} \phi_{i}(i=1,2, \ldots, N / 2),
$$

where

$$
H^{\mathrm{HF}}=h+\sum_{j=1}^{N / 2}\left(2 J_{j}-K_{j}\right),
$$

where $J_{j}(1)$ is the Coulomb potential at position $r_{1}$ due to charge density $\left|\phi_{j}\right|^{2}$,

$$
J_{j}(1)=\int d^{3} r_{2} \frac{\phi_{j}^{*}(2) \phi_{j}(2)}{r_{12}},
$$

and where $K_{j}$ is the exchange operator defined so that

$$
K_{j} \phi_{i}(1)=\phi_{j}(1) \int d^{3} r_{2} \frac{\phi_{j}^{*}(2) \phi_{i}(2)}{r_{12}} .
$$

The HF equations (8) are solved iteratively and (upon convergence) the final self-consistent orbitals are used to evaluate the total energy, Eq. (7). This provides a total energy at just the one geometry and hence this whole procedure must be repeated for a sequence of geometries to determine the geometry of the molecule or a potential surface for a reaction.

From Eq. (8), the eigenvalue $\epsilon_{i}$ in (8a) has the form

$$
\epsilon_{i}=h_{i i}+\sum_{j}\left(2 J_{i j}-K_{i j}\right)
$$

which corresponds to the negative of the energy increase that would result if one electron in orbital $\phi_{i}$ were deleted (ionized) from wavefunction (1). Use of this approximation to the ionization potential is often referred to as the Koopmans Theorem. Summing over all occupied eigenvalues (11) (with a factor of two since there are two electrons in each spatial orbital) leads to

$$
\begin{gathered}
\sum_{i=1}^{N / 2} 2 \epsilon_{i}=\sum_{i} 2 h_{i i}+\sum_{i, j}\left(4 J_{i j}-2 K_{i j}\right) \\
=E_{1}+2 E_{2} .
\end{gathered}
$$

Thus, comparing with Eq. (7), the total energy is

$$
E=\sum_{i} 2 \epsilon_{i}+\left[E_{0}-E_{2}\right] .
$$

That is, from a knowledge of orbital eigenvalues alone, one cannot calculate the total energy. In addition to the $\left\{\epsilon_{i}\right\}$, one must be able to separately evaluate the one- and two-electron energy terms. Since total energies are required to determine geometries, one cannot calculate geometries without separate evaluation of the one- and two-electron energy terms.

\section{Basis sets}

The HF operator $H^{\mathrm{HF}}$ involves integral operators $\left(K_{j}\right)$, second-order partial derivatives $\left(-1 / 2 \nabla^{2}\right)$, and complicated functions of electronic coordinates $\left(J_{j}\right)$. As a result, a direct solution of these equations is practical only for atoms in which symmetry can be used to remove all but the radial coordinates. For molecular systems (8) is solved by expanding each (unknown) molecular orbital $\phi_{i}$ in terms of a known (finite) set of basis functions

$$
\begin{gathered}
\left\{\chi_{\mu} ; \mu=1,2, \ldots, P\right\} \\
\phi_{i}=\sum_{\mu=1}^{P} \chi_{\mu} C_{\mu i} i=1,2, \ldots, N / 2 .
\end{gathered}
$$

This converts the coupled nonlinear integro-differential Eq. 
(8) to coupled nonlinear algebraic Eqs. (16),

$$
\sum_{\nu=1}^{P} H_{\mu \nu}^{\mathrm{HF}} C_{\nu i}=\sum_{\nu=1}^{P} S_{\mu \nu} C_{\nu i} \epsilon_{i} \begin{aligned}
& \mu=1,2, \ldots, P \\
& i=1,2, \ldots, N / 2
\end{aligned}
$$

where

$$
S_{\mu \nu}=\left\langle\chi_{\mu} \mid \chi_{\nu}\right\rangle
$$

and

$$
\begin{aligned}
H_{\mu \nu}^{\mathrm{HF}}=\left\langle\chi_{\mu}\left|H^{\mathrm{HF}}\right| \chi_{\nu}\right\rangle= & \left\langle\chi_{\mu}|h| \chi_{\nu}\right\rangle \\
& +\sum_{j=1}^{N / 2}\left\langle\chi_{\mu}\left|\left(2 J_{j}-K_{j}\right)\right| \chi_{\nu} \chi .\right.
\end{aligned}
$$

Expanding $\phi_{j}$ in terms of the basis (14) the latter terms of Eq. (16c) become

$$
\begin{aligned}
\left\langle\chi_{\mu}\right|\left(2 J_{j}-\right. & \left.K_{j}\right)\left|\chi_{\nu}\right\rangle \\
& =\sum_{\sigma, \eta}\left[2\left(\chi_{\mu} \chi_{\nu} \mid \chi_{\sigma} \chi_{\eta}\right)-\left(\chi_{\mu} \chi_{\sigma} \mid \chi_{\nu} \chi_{\eta}\right)\right] D_{\sigma \eta},
\end{aligned}
$$

where

$$
\left(\chi_{\mu} \chi_{\nu} \mid \chi_{\sigma} \chi_{\eta}\right) \equiv\left\langle\chi_{\mu}(1) \chi_{\sigma}(2)\left|1 / r_{12}\right| \chi_{\nu}(1) \chi_{\eta}(2)\right\rangle,
$$

and where

$$
D_{\sigma \eta}=\sum_{j=1}^{N / 2} C_{\sigma j}^{*} C_{\eta j}
$$

(the density matrix).

For a complete basis $(P=\infty)$ in Eq. (14), the solution of the HF matrix Eq. (16) is equivalent to solving the HF Eq. (7). For practical calculations it was necessary to learn how to choose the smallest basis (14) so as not to bias the resulting calculations. In addition, it was necessary to use basis functions so that the resulting integrals in Eqs. (16) and (17) can be evaluated efficiently. Much of the quantum chemistry activities in the 1960's was devoted to developing increasingly efficient computer programs for evaluating the integrals and to exploring the effect of size and type of basis on molecular geometries and properties.

It is now generally agreed that:

(a) The optimal basis functions for integral evaluation ${ }^{7,9}$ are Cartesian Gaussian orbitals of the form

$$
\chi\left(x_{A}, y_{A}, z_{A}\right)=N x_{A}^{m} y_{A}^{p} z z^{q} e^{-\alpha r^{2} A},
$$

where all coordinates are defined with respect to center $A$ (generally the position of a nucleus) and $N$ is a normalization factor. Defining $\ell=m+p+q$, these functions are referred to as $s, p, d, f, \ldots$ for $\ell=0,1,2,3, \ldots$; however, the six functions for $\ell=2$ are a linear combination of $s$ and $d$ functions, and the ten functions for $\ell=3$ are a linear combination of $p$ and $f$ functions.

(b) Although primitive Gaussian functions such as Eq. (20) are quite adequate for describing the shapes of molecular orbitals in the bond regions, they are not adequate for describing the cusps that occur near nuclear centers. Thus, to describe the inner parts of $1 s, 2 p, 3 d$, and $4 f$ orbitals, we must use a number of primitive Gaussians (20). However, it was found that for various geometries and various molecules the Gaussian describing the core region of a specific atom could be grouped together into one contracted function having the whole set of Gaussians combined with fixed relative coefficients, ${ }^{6,7,9}$

$$
\bar{\chi}_{\text {contracted }}=\sum_{\mu} d_{\mu} \chi_{\mu, \text { prim }} .
$$

Thus, in calculating the wavefunctions (e.g., HF), the contracted basis functions are used, reducing the size of the matrices to be handled in the iterative calculations; however, integrals are evaluated in terms of primitive functions (20) and immediately combined to form integrals over the contracted functions (21).

(c) With an appropriate strategy for choosing the contracted basis functions, one can choose a basis from atomic calculations that will be adequate for essentially all molecular calculations involving that atom. The smallest generally adequate basis set involves (i) one contracted function for each core orbital (e.g., $1 s$ for C; $1 s, 2 s, 2 p, 3 s, 3 p, 3 d$ for Ge), (ii) two contracted functions for each valence orbital (e.g., $2 s, 2 p$ for $\mathrm{C} ; 4 s, 4 p$ for $\mathrm{Ge}$ ), and (iii) one set of higher angular momentum functions suitable for polarizing the valence orbitals (e.g., $p$ functions for $H, d$ functions for $\mathrm{C}$ or $\mathrm{Ge}$ ).

In the jargon of quantum chemists, the basis in (c) is referred to as double zeta (or valence double zeta) plus polarization functions and denoted as DZd. If the polarization functions are deleted (adequate for many purposes), the basis is referred to as double zeta or DZ. If only one contracted function is used for each valence orbital [part (ii) of (c)], the resulting basis is referred to as minimum basis set or MBS. An alternative (more specific and more informative) nomenclature used by Pople and co-workers ${ }^{7}$ denotes MBS as STO$3 \mathrm{G}, \mathrm{DZ}$ as $4-31 \mathrm{G}$, and DZd as 6-31G*. Parameters for such basis sets have been published. ${ }^{7,9}$

\section{Accuracy}

Using such basis sets, HF calculations have been carried out on hundreds of molecules (including radicals and ions) $)^{7,10}$ and generally lead to bond lengths accurate to 0.01 to $0.02 \hat{A}$ and bond angles accurate to $1^{\circ}$ to $3^{\circ}$. Such calculations have been of great use to chemists since many radicals and ions are too short-lived for experimental geometry determinations. The barriers to rotations about single bonds are small ( 0.05 to 0.2 $\mathrm{eV}$ ) but are accurately predicted by these methods (to about 10\%). ${ }^{11}$ Global properties such as dipole moments are given quite accurately (to about $0.02 e \AA$ ); however, point properties such as spin density at the nucleus are not accurate at this level. ${ }^{12}$

In addition to these quantitative successes, this method is conceptually simple and provides the basis for qualitative rules of chemical reactions and spectra of atoms, molecules, inorganic complexes, and solids. However, there are some serious problems with $\mathrm{HF}$ theory that are of great significance for studies of surfaces. For example, HF leads to very unreliable bond energies, as illustrated in Table $I_{.}{ }^{13}$

The basic problem with the HF description of bonds is that cleavage of a covalent bond generally leads to two radicals (with the two electrons of the bond each separating onto individual fragments), whereas the $\mathrm{HF}$ wavefunction, having only doubly-occupied orbitals, leads perforce to mixtures of covalent and ionic products. This is discussed further in Sec. 
TABLE I. Bond energies (eV). These values are from the bottom of the potential curve $\left(D_{e}\right)$ and hence the zero-point energy must be subtracted to yield the direct experimental value. All calculations use a DZd basis set. (See Ref. 13).

\begin{tabular}{lccc}
\hline \hline & & GVB-Cl & Experimental \\
\hline & & & \\
$\mathrm{CH}_{3}-\mathrm{CH}_{3}$ & 3.13 & 4.07 & 4.19 \\
$\mathrm{CH}_{3}-\mathrm{OH}$ & 2.73 & 4.25 & 4.28 \\
$\mathrm{HO}-\mathrm{OH}$ & 0.04 & 2.45 & 2.26 \\
$\mathrm{H} 2 \mathrm{C}=\mathrm{CH}$ & 5.35 & 7.44 & 7.82 \\
$\mathrm{H}_{2} \mathrm{C}=\mathrm{O}$ & 4.58 & 7.57 & 7.93 \\
$\mathrm{O}_{2}$ & & 4.26 & 4.23 \\
\hline \hline
\end{tabular}

II. D. Some surfaces [e.g., $\mathrm{Si}(111), \mathrm{Si}(100)]$ lead to electronic states similar to those occurring as chemical bonds are broken. As a result, $\mathrm{HF}$ theory is expected to lead to serious problems for such states, as discussed in Sec. III.

\section{B. Generalized valence bond}

In the simple valence bond (VB) description, one starts with the proper atomic orbitals of the fragments and pairs these orbitals on different centers to form the bonds. For example, for $\mathrm{H}_{2}$ the $\mathrm{VB}$ spatial wavefunction is ${ }^{14}$

$$
\Phi^{\mathrm{VB}}(1,2)=\chi_{\ell}(1) \chi_{r}(2)+\chi_{r}(1) \chi_{\ell}(2),
$$

and the total wavefunction is

$$
\Psi^{\mathrm{VB}}(1,2)=\Psi^{\mathrm{VB}}(1,2)[\alpha(1) \beta(2)-\beta(1) \alpha(2)],
$$

where $\chi_{\ell}$ and $\chi_{r}$ are atomic ls functions centered on the two protons. This wavefunction is exact for an infinite internuclear distance but only approximate for finite separations. Unfortunately, the errors in calculated bond distances and bond lengths are too large for quantitative purposes.

The GVB method ${ }^{3,15}$ is a self-consistent field version of VB in which one allows each bond pair to be described in terms of two different orbitals as in VB but optimizes the shape of the orbitals as in HF. Thus, for $\mathrm{H}_{2}$ the GVB wavefunction has the form

$$
\Psi^{\mathrm{GVB}}(1,2)=\phi_{a}(1) \phi_{b}(2)+\phi_{b}(1) \phi_{a}(2),
$$

where the total wavefunction is

$$
\begin{gathered}
\Psi^{\mathrm{GVB}}(1,2)=\Phi^{\mathrm{GVB}}(1,2)[\alpha(1) \beta(2)-\beta(1) \alpha(2)] \\
=a\left[\left(\phi_{a} \phi_{b}+\phi_{b} \phi_{a}\right)(\alpha \beta)\right] \\
=a\left[\left(\phi_{a} \alpha\right)\left(\phi_{b} \beta\right)\right]+a\left[\left(\phi_{b} \alpha\right)\left(\phi_{a} \beta\right)\right] .
\end{gathered}
$$

Thus the GVB wavefunction is a superposition of more than one Slater determinant.

Evaluating the total energy of (23) in terms of $\phi_{a}$ and $\phi_{b}$ and applying the variational principle to obtain the optimum orbitals (the GVB orbitals) leads to a set of equations similar to the $\mathrm{HF}$ equations (8) except that there is now one equation for each orbital. These equations are solved using a basis set expansion (14), and the computational bottlenecks are similar to those in $\mathrm{HF}^{3,15(\mathrm{~b})}$ For $\mathrm{H}_{2}$ the optimum orbitals lead to $\phi_{a}$ more localized on the left side of the molecule and $\phi_{b}$ more localized on the right side. For $R=\infty$, the optimum GVB orbitals are just atomic orbitals leading to the VB wavefunc- tion. Forcing $\phi_{a}$ to be equal to $\phi_{b}$ leads to the HF wavefunction so that HF is a special case of GVB.

For a many-electron system, the GVB wavefunction can generally be obtained from HF by replacing each electron pair

$$
\phi_{i}(2 i-1) \phi_{i}(2 i)
$$

of the $\mathrm{HF}$ wavefunction by the electron pair

$$
\phi_{i a}(2 i-1) \phi_{i b}(2 i)+\phi_{i b}(2 i-1) \phi_{i a}(2 i),
$$

leading to a total wavefunction of the form

$$
a\left[\left(\phi_{1 a} \phi_{1 b}+\phi_{1 b} \phi_{1 a}\right)\left(\phi_{2 a} \phi_{2 b}+\phi_{2 b} \phi_{2 a}\right) \ldots(\alpha \beta \alpha \beta \ldots)\right] .
$$

Note that although all orbitals are allowed to be different, the total wavefunction is a proper eigenstate of total spin and satisfies the Pauli principle (expansion of this wavefunction would lead to numerous Slater determinants).

For the systems discussed in this paper, the GVB orbitals are each primarily localized on a single center and hence the description is qualitatively as in VB.

\section{GVB-CI}

In obtaining sufficient accuracy for assessing reaction mechanisms, it is necessary to go beyond the GVB wavefunction. In particular, there are effects involving simultaneous correlation of the motion of electrons in adjacent bonds that have a large effect on bond energies. The reason is that these effects generally become small as one of the bonds is broken and hence the bond energy is systematically low if all such terms are excluded. These effects lead to terms in the wavefunction in which electrons of several bond pairs are simultaneously excited to the correlating orbitals of the bond pairs. The resulting wavefunction is referred to as GVB-CI ("CI" denotes configuration interaction or superposition of configurations). The results of several GVB-CI calculations ${ }^{13}$ are tabulated in Table I.

\section{Illustrations for two-electron systems}

The basic problem with the $\mathrm{HF}$ description of bonds can be illustrated with the case of two electrons. [In the discussion we use only two basis functions, $\chi_{\ell}$ and $\chi_{r}$; this is greatly oversimplified but serves to illustrate the basic points.] Letting $\phi_{g}$ and $\phi_{u}$ be the bonding and antibonding orbitals,

$$
\begin{aligned}
\phi_{g} & =\chi_{\ell^{-}}+\chi_{r} \\
\phi_{u} & =\chi_{\ell}-\chi_{r}
\end{aligned}
$$

(ignoring normalization), where $\chi_{\ell}$ and $\chi_{r}$ are localized on the left and right centers, the HF wavefunction is

$$
a\left\{\left[\phi_{g}(1) \alpha(1)\right]\left[\phi_{g}(2) \beta(2)\right]\right\}=\Phi^{H F}(1,2)[\alpha \beta-\beta \alpha],
$$

where the spatial part of the wavefunction is

$$
\begin{gathered}
\Phi^{\mathrm{HF}}(1,2)=\phi_{g}(1) \phi_{g}(2)=\left[\chi_{\ell}(1)+\chi_{r}(1)\right]\left[\chi_{\ell}(2)+\chi_{r}(2)\right] \\
=\left[\chi_{\ell}(1) \chi_{\ell}(2)+\chi_{r}(1) \chi_{r}(2)\right] \\
+\left[\chi_{\ell}(1) \chi_{r}(2)+\chi_{r}(1) \chi_{\ell}(2)\right] \\
=\Phi_{\text {ion }}+\Phi_{\text {cov }}
\end{gathered}
$$

That is, the HF description forces an equal amount of covalent 
and ionic character. For the case of a strong bond, the overlap of $\chi_{\ell}$ and $\chi_{T}$ is large (say, $S=0.7$ ), leading to an overlap between (renormalized) $\Phi_{\text {ion }}$ and $\Phi_{\text {cov }}$ of $\left[2 S /\left(1+S^{2}\right)\right] \approx 0.94$. In this case, the restriction of a fixed ratio of $\Phi_{\text {ion }}$ to $\Phi_{\text {cov }}$ is not of major importance. However, for small overlaps, $\Phi_{\text {ion }}$ and $\Phi_{\text {cov }}$ lead to very different energies and hence the restriction to a fixed ratio can cause severe problems. This occurs as bonds are broken and it also occurs in other systems that would naturally have weakly overlapping orbitals.

In contrast, the GVB wavefunction (for this simple case) ${ }^{16}$ has the form

$$
\Phi^{\mathrm{GVB}}=\phi_{\ell}(1) \phi_{r}(2)+\phi_{r}(1) \phi_{\ell}(2)
$$

where

$$
\begin{gathered}
\phi_{\ell}=\chi_{\ell}+\lambda \chi_{r} \\
\phi_{r}=\chi_{r}+\lambda \chi_{\ell}
\end{gathered}
$$

(again ignoring normalization), where the coefficient $\lambda$ is optimized. Substituting (27) into (26) then leads to

$$
\Phi^{\mathrm{GVB}}=\left(1+\lambda^{2}\right) \Phi_{\mathrm{cov}}+2 \lambda \Phi_{\text {ion }} .
$$

Thus in GVB the ratio of ionic to covalent character is optimized. For cases with small overlap, the optimum $\lambda$ is very small, leading to a simple valence bond wavefunction. For cases with large overlap, both terms are important $(\lambda \sim$ $0.1)$.

An alternative form of the GVB wavefunction is obtained by substituting the molecular orbitals from (24) into (26). This leads to

$$
\Phi^{\mathrm{GVB}}=c_{1} \phi_{g}(1) \phi_{g}(2)-c_{2} \phi_{u}(1) \phi_{u}(2) .
$$

In molecular orbital language, $\phi_{u}$ is the orbital correlating the motions of the electron in the $\phi_{g}$ orbital, and the GVB wavefunction can be viewed as simultaneous optimization of both the occupied $\left(\phi_{g}\right)$ and correlating $\left(\phi_{u}\right)$ orbitals.

\section{E. Approximate methods}

Until recent years, ab initio (i.e., first principles) calculations were impractical except on small molecules, and a number of more approximate methods have been developed. Most of these methods are approximations to the HF wavefunction and most involve an approximation to the MBS form of the $\mathrm{HF}$ wavefunction.

The simplest method is extended Hückel theory ${ }^{17}$ (EHT) in which the elements of the HF matrix (13b) are derived empirically,

$$
\begin{gathered}
H_{\mu \mu}^{\mathrm{HF}}=\alpha \\
H_{\mu \nu}^{\mathrm{HF}}=\beta_{\mu \nu},
\end{gathered}
$$

if $\mu$ and $\nu$ are on adjacent (bonded) atoms, and

$$
H_{\mu \nu}^{\mathrm{HF}}=0
$$

otherwise. The $\alpha$ is set equal to an atomic ionization potential and the resonance integral, $\beta$, is set proportional to the overlap of the atomic functions. This method is not self-consistent since the two-electron interactions are implicitly built into the $\alpha$ and $\beta$ parameters. The analogous approach in band theory is referred to as the tight binding method. ${ }^{18}$ An iterative method ${ }^{19}$ in which $\alpha$ is adjusted by the total calculated population on a center is sometimes used.

Since the one- and two-electron terms are combined in the parameters, it is not possible to separate these quantities in order to calculate total energies and hence geometries. The total energy has been approximated as

$$
E \approx \sum_{i} 2 \epsilon_{i},
$$

leading to some spectacular failures. For example, the calculated CC bond lengths for acetylene, ethylene, benzene, and ethane are $0.87,1.52,1.57$ and $2.06 \AA$, respectively, whereas the experimental values are $1.21,1.34,1.39$, and 1.54 $\AA$, respectively. ${ }^{20}$ Even worse, it was found that benzene decomposes into three acetylenes ${ }^{20}$<smiles>C=CC=CC=C</smiles>

(downhill by $11.2 \mathrm{eV}$ with not even a barrier!), despite the fact that experimentally benzene is $6.2 \mathrm{eV}$ more stable than three acetylenes. A problem with extension of EHT to atoms past the first (carbon) row of the periodic table has been the lack of sufficient experimental data to determine values for the parameters.

A significant improvement of EHT was the CNDO (and INDO) series of approximations ${ }^{21}$ in which dominant twoelectron interactions, all one- and two-center Coulomb terms

\section{$\left(\chi_{\mu} \chi_{\mu} \mid \chi_{\nu} \chi_{\nu}\right)$}

were evaluated exactly and $\alpha$ and $\beta$ parameters were empirically chosen to represent other terms. This approach generally leads to bond lengths within $0.1 \AA$ and bond energies within $5 \mathrm{eV} .{ }^{21}$ By empirically fitting these parameters to numerous experimental values for energies and geometries, Dewar and co-workers ${ }^{22}$ have developed a series of programs $\mathrm{MINDO} / \mathrm{N}$ useful in estimating energies and geometries.

Essentially all band calculations on solids are based on wavefunctions having the same form as closed-shell $\mathrm{HF}$. Thus the problems with HF in describing weakly overlapping singly occupied orbitals should apply also to band calculations. Of course, bulk solids do not generally have such singly occupied orbitals (except for defects and also certain transition metal systems). However, similar techniques are also used for systems with surfaces, and problems could occur here. A second serious difficulty with normal band calculations is that is is not possible to obtain total energies; thus one cannot calculate geometric structure. Recently, Chadi has proposed an empirical approach to getting total energies. ${ }^{23}$ The idea is to get $\Sigma_{\mathrm{i}} 2 \epsilon_{\mathrm{i}}$ from the band calculation and to replace $\left(E_{0}-E_{2}\right)$, see Eq. (13), with an empirical harmonic function in the particle coordinates. The coefficients of the latter function are obtained so that the total energy function, $E$ leads to experimental geometries and force constants for the bulk system. For studies of surfaces these functions are then added to $\Sigma 2 \epsilon_{i}$ to obtain a total energy function. A potential problem here is that the hybridization and density distribution around the surface atoms is different from the bulk so that the empirical force function may not apply to the surface atoms. 
A quite different approach to calculating wavefunctions is the muffin-tin scattered-wave $X \alpha$ approach. ${ }^{24,25}$ This is described in detail by Messmer ${ }^{25}$ and we will add only a few comments. The wavefunction is taken to be of the same form as in HF, say (1) for a closed-shell wavefunction. However the exchange term

$$
V_{x}=-\sum_{j=1}^{N / 2} K_{j}
$$

of Eq. (8b) is written as

$$
V_{x} \cong-\alpha(81 / 8 \pi)^{1 / 3} \rho^{1 / 3},
$$

where $\alpha \approx 0.7$. In addition, the potential is generally taken to be of muffin-tin form, i.e., spherically symmetric within a sphere about each nucleus and constant in between the spheres. Although the wavefunction is of HF form as in (1), the proponents of this method generally argue that it includes some electron correlation effects. An advantage of this method is that the two-electron interactions are expressed in terms of densities, allowing a heavy atom such as $\mathrm{Pt}$ to be studied as simply as a small atom such as $\mathrm{Ni} \mathrm{A}$ serious problem is that because of the use of the muffin-tin approximation, the total energies are very inaccurate, leading to very poor potential surfaces. For example $\mathrm{H}_{2} \mathrm{O}$ is calculated ${ }^{26 a}$ to be linear in $\mathrm{X} \alpha$ (the experimental bond angle is $104.5^{\circ}$ ), and $\mathrm{NH}_{3}$ is calculated ${ }^{26 \mathrm{~b}}$ to be planar (experimentally $\mathrm{NH}_{3}$ is pyramidal with a bond angle of $\left.107 \cdot 6^{\circ}\right)$. Experimentally, the planar form of $\mathrm{NH}_{3}$ is $0.2 \mathrm{eV}$ higher in energy, ${ }^{11}$ whereas muffin-tin $\mathrm{X} \alpha$ leads to the planar geometry $1.4 \mathrm{eV}$ lower than the pyramidal! ${ }^{26 \mathrm{~b}}$ On the other hand, application of the muffin-tin $X \alpha$ method to ozone ${ }^{27}$ led to better results than ab initio HF (but worse than ab initio GVB). ${ }^{28}$

\section{DISCUSSION}

We will illustrate some features and capabilities of some of these theoretical methods with several examples.

\section{A. Oxygen molecule}

As discussed in more detail elsewhere, ${ }^{29-31}$ the VB description of $\mathrm{O}_{2}$ leads to configurations such as

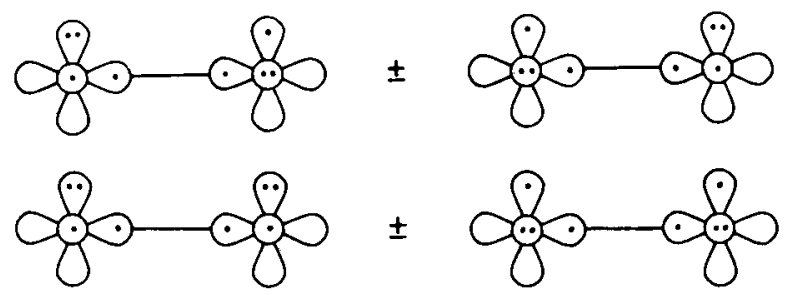

where only the $p$ orbitals are shown (both directions perpendicular to the axis), and the dots indicate the number of electrons in each orbital (the $1 s$ and $2 s$ pairs have been omitted). As the molecule is pulled apart, the wavefunction changes smoothly to that of two oxygen atoms, as shown in Fig. 1.29

The $\mathrm{HF}$ wavefunction for $\mathrm{O}_{2}$ has the configuration

$$
\begin{aligned}
\left(1 \sigma_{g} 1 s\right)^{2}\left(1 \sigma_{u} 1 s\right)^{2}\left(2 \sigma_{g} 2 s\right)^{2} & \\
\times & \left(2 \sigma_{u} 2 s\right)^{2}\left(3 \sigma_{g} 2 p\right)^{2}\left(1 \pi_{u} 2 p\right)^{4}\left(1 \pi_{g} 2 p\right)^{2},
\end{aligned}
$$

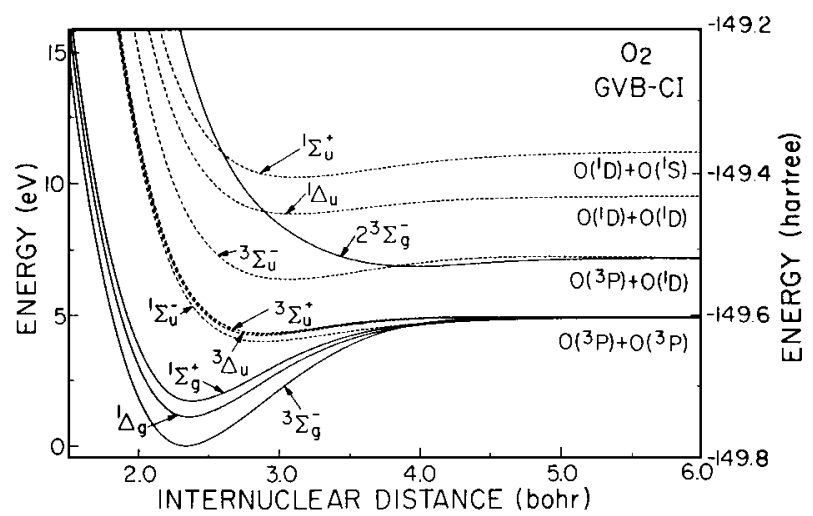

FIG. 1. Potential curves for low-lying states of $\mathrm{O}_{2}$ (from GVB-CI calculations $^{29}$ ).

where the latter three orbital sets (with eight electrons) correspond closely to the eight electrons indicated in (31). In this case, dissociation leads to incorrect potential curves, as indicated in Fig. 2.

Using the GVB orbitals and carrying out a CI within these orbitals leads to accurate potential curves; in Table II we compare bond distances, vibrational frequencies, and bond energies in order to provide some idea of the accuracy.

\section{B. Ozone molecule}

The VB description of ozone $\left(\mathrm{O}_{3}\right)$ is obtained by combining an $\mathrm{O}$ atom to a diagram such as (31), leading to diagrams such as $^{15 a}$

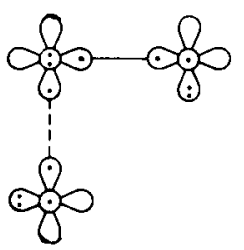

The set of low-lying states so obtained is shown in Fig. 3 and denoted as $4 \pi, 5 \pi$, or $6 \pi$ to indicate how many electrons are in $p$ orbitals perpendicular to the molecular plane. In each case there is a singly-occupied orbital on each terminal atom. In the ground state these singly-occupied orbitals are singlet-paired, leading to a weak bonding effect (the doubly occupied $p \pi$ orbital on the central $\mathrm{O}$ plays a major factor in

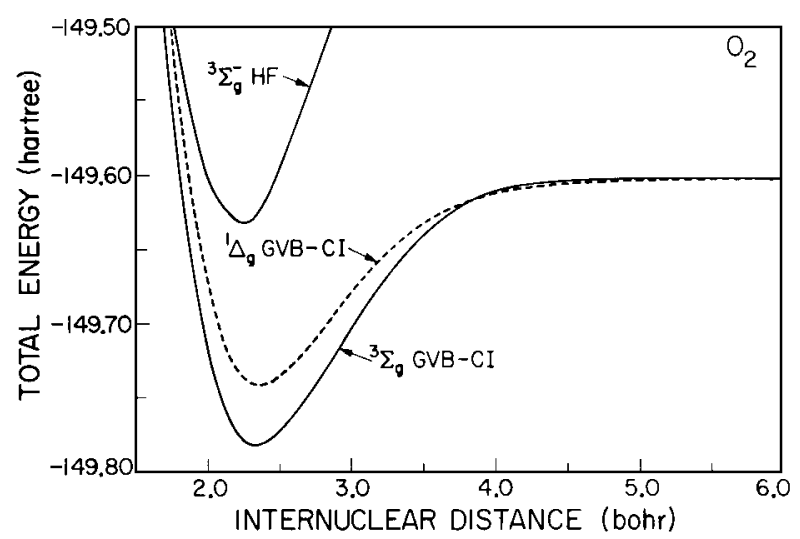

FIG. 2. HF potential curve for the ground state $\left({ }^{2} \Sigma_{g}^{-}\right)$of $\mathrm{O}_{2}$ compared with the GVB-CI potential curve for the lowest two states. ${ }^{29}$ 
TABLE II. Spectroscopic Constants for $\mathrm{O}_{2}$

\begin{tabular}{|c|c|c|c|c|c|c|c|c|}
\hline & \multicolumn{2}{|c|}{$\begin{array}{l}\text { Excitation } \\
\text { energies } \\
\text { (eV) }\end{array}$} & \multicolumn{2}{|c|}{$\begin{array}{c}\text { Bond distance } \\
(\AA)\end{array}$} & \multicolumn{2}{|c|}{$\begin{array}{c}\text { Vibrational } \\
\text { frequency } \\
\text { (meV) }\end{array}$} & \multicolumn{2}{|c|}{$\begin{array}{l}\text { Bond } \\
\text { energyc } \\
(\mathrm{eV})\end{array}$} \\
\hline & Calculated $^{d}$ & Experimental ${ }^{\mathrm{a}}$ & Calculated ${ }^{d}$ & Experimentala & Calculated $\mathrm{d}^{\mathrm{c}, \mathrm{d}}$ & Experimental ${ }^{a}$ & Calculated $^{d}$ & Experimentala \\
\hline $1 \Sigma_{u}^{+}$ & 10.19 & & 1.655 & & 81 & & $0.92^{\mathrm{b}}$ & \\
\hline$\Delta_{u}^{u}$ & 8.79 & & 1.648 & & 78 & & $0.56^{b}$ & \\
\hline$B^{3} \Sigma_{u}^{-}$ & 6.31 & 6.17 & 1.625 & 1.604 & 83 & 88 & $0.81^{\mathrm{b}}$ & 1.01 \\
\hline$A^{3} \Sigma_{u}^{+}$ & 4.25 & 4.39 & 1.528 & 1.522 & 104 & 99 & 0.63 & 0.82 \\
\hline$C^{3} \Delta_{u}^{u}$ & 4.17 & 4.31 & 1.522 & & 106 & & 0.70 & 0.91 \\
\hline$c^{\prime} \Sigma_{u}^{-}$ & 3.94 & 4.10 & 1.525 & 1.517 & 103 & 98 & 0.94 & 1.12 \\
\hline$b^{1} \Sigma_{g}^{+}$ & 1.69 & 1.64 & 1.260 & 1.227 & 187 & 178 & 3.19 & 3.58 \\
\hline$a^{1} \Delta_{g}^{g}$ & 1.09 & 0.98 & 1.249 & 1.216 & 198 & $\sim 187$ & 3.79 & 4.23 \\
\hline$X^{3} \Sigma_{g}^{-}$ & 0 & 0 & 1.238 & 1.208 & 210 & 196 & 4.88 & 5.21 \\
\hline
\end{tabular}

a P. Krupenie, J. Phys. Chem. Ref. Data 1, 423 (1972).

b These states dissociate to an excited state on one of the $\mathrm{O}$ atoms.

${ }^{c}$ Evaluated at the minimum on the potential curve.

d Reference 29.

coupling the singly occupied orbitals, an effect analogous to superexchange coupling in antiferromagnetic transition metal oxides).

Quantitative studies based on the GVB wavefunctions have explained some confusing spectroscopic results and guided experimental probes on the excited states. ${ }^{15 a, 28,31}$ As indicated in Table III, such theoretical calculations can provide a high level of accuracy.

In Table IV we compare the excitation energies from GVB-CI calculations with those from $\mathrm{HF}$ calculations. It is apparent that there are very serious errors in the HF description. Most serious is that although ozone is known to have a singlet ground state, HF predicts a ground state triplet (1 ${ }^{3} B_{2}$ ) and puts the lowest singlet state at over $2 \mathrm{eV}$ above this triplet state (another triplet state $2^{3} B_{2}$ is also placed below the first singlet). The problem with HF is obvious from the VB diagram (32). The two singly occupied orbitals are separated by a large distance and have a low overlap. This is analogous to an $\mathrm{H}_{2}$ molecule at large $R$. The triplet coupling of the orbital is well described by HF (leading to the ${ }^{3} B_{2}$ state of ozone), but the singlet coupling (leading to the $1^{1} A_{1}$ state) is

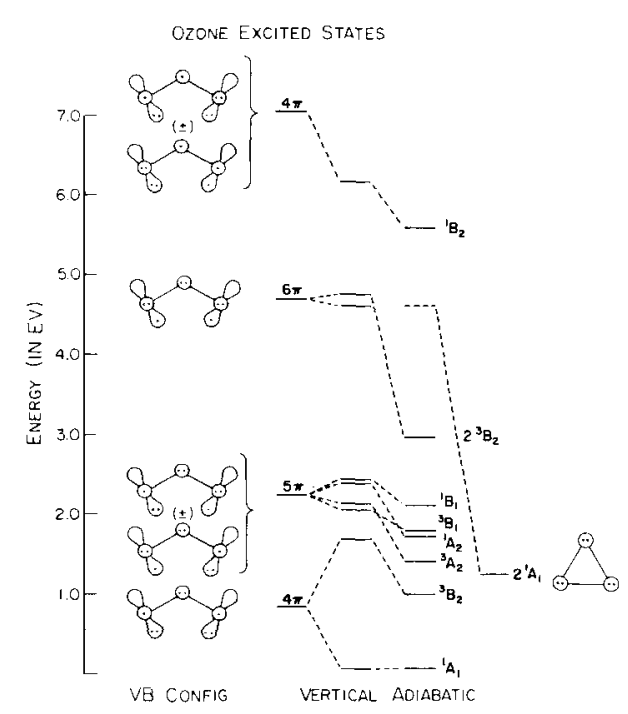

FIG. 3. The electronic excited states of ozone. ${ }^{28,32}$ not. As a result, HF interchanges the states. As indicated below, some surfaces lead to analogous electronic states and hence similar problems.

Given the serious problems that HF has with ozone, one would suspect that muffin-tin $X \alpha$ would lead to similar problems (since the form of the wavefunction is similar). In fact, however, the $X \alpha$ calculations lead to the correct ground state, ${ }^{27}$ providing evidence that these calculations do include some electron correlation effects (as often suggested by $\mathrm{X} \alpha$ proponents). Even so, there are serious discrepancies between the $X \alpha$ results and the accurate GVB-CI calculations, suggesting that $X \alpha$ is missing some very important electron correlation effects. A serious problem at this point is how to proceed from $X \alpha$ to accurate results. If part of the electron correlation is included in $X \alpha$, how does one include the missing electron correlation effects? An advantage of ab initio methods such as GVB is that there is a well-defined procedure for adding additional correlation effects, eventually leading to essentially exact results.

\section{The (111) surface of $\mathrm{Si}$}

The unreconstructed $(1 \times 1)$ surface of Si contains an array of singly-occupied orbitals (dangling-bond orbitals) separated from each other by (at least) one subsurface atom. Consider just one pair of such dangling bond orbitals,

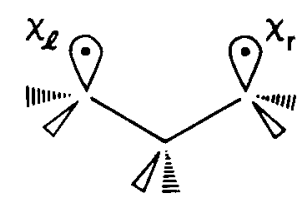

TABLE III. Spectroscopic constants for ozone. ${ }^{a}$

\begin{tabular}{lcc}
\hline \hline & GVB.CI & Experimental \\
\hline O-O bond length $(\AA)$ & $1.299 \AA$ & $1.278 \AA$ \\
OOO bond angle (degrees) & $116.0^{\circ}$ & $116.8^{\circ}$ \\
$\nu_{1}(\mathrm{meV})$ & 153 & 138 \\
$\nu_{3}(\mathrm{meV})$ & 87.7 & 87.4 \\
\hline \hline
\end{tabular}

Reference 28. 
TABLE IV. Excitation energies (eV) for ozone (based on ground state geometry). ${ }^{\mathrm{C}}$

\begin{tabular}{lcccc}
\hline \hline & GVB-CI & $\mathrm{HF}^{\mathrm{a}}$ & $\mathrm{X} \alpha-\mathrm{SW}^{\mathrm{b}}$ & \\
\hline $1^{1} A_{1}$ & $4 \pi$ & -1.60 & +2.16 & -1.65 \\
$1^{3} B_{2}$ & $4 \pi$ & 0 & 0 & 0 \\
$1^{3} A_{2}$ & $5 \pi$ & 0.49 & 2.95 & -0.53 \\
$1^{3} B_{1}$ & $5 \pi$ & 0.41 & 2.74 & -0.43 \\
$1^{1} A_{2}$ & $5 \pi$ & 0.74 & 3.41 & 0.82 \\
$1^{1} B_{1}$ & $5 \pi$ & 0.81 & 3.58 & 1.00 \\
$2^{3} B_{2}$ & $6 \pi$ & 1.57 & 1.64 & 1.66 \\
$2^{1} A_{1}$ & $6 \pi$ & 1.98 & 5.88 & 2.77 \\
$1^{1} B_{2}$ & $4 \pi$ & 4.52 & 6.01 & 1.34 \\
\hline \hline
\end{tabular}

a Reference 28 .

b Reference 27.

c The states are referenced with respect to $1{ }^{3} B_{2}$ since all wavefunctions lead to a good description of this state.

in which two singly-occupied orbitals are separated by an intervening atom so that their overlap is small.

In the molecular orbital description the orbitals are ${ }^{14}$

$$
\begin{aligned}
& \phi_{g}=\chi_{\ell}+\chi_{r} \\
& \phi_{u}=\chi_{\ell}-\chi_{r},
\end{aligned}
$$

with a small separation in the orbitals (to be specific, we will assume $\phi_{g}$ to be lower than $\phi_{u}$ ). The molecular orbital wavefunctions for the two-electron system are

$$
\begin{gathered}
{ }^{\mathrm{I}} \Psi_{g 1}^{\mathrm{MO}}=\phi_{g}(1) \phi_{g}(2)[\alpha \beta-\beta \alpha] \\
{ }^{\mathrm{I}} \Psi_{g 2}^{\mathrm{MO}}=\phi_{u}(1) \phi_{u}(2)[\alpha \beta-\beta \alpha] \\
{ }^{3} \Psi_{u}^{\mathrm{MO}}=\left[\phi_{g}(1) \phi_{u}(2)-\phi_{u}(1) \phi_{g}(2)\right] \alpha \alpha \\
{ }^{\mathrm{I}} \Psi_{u}^{\mathrm{MO}}=\left[\phi_{g}(1) \phi_{u}(2)+\phi_{u}(1) \phi_{g}(2)\right][\alpha \beta-\beta \alpha] .
\end{gathered}
$$

Substituting (33) into (34)-(37) and omitting the spin factors leads to

$$
\begin{aligned}
&{ }^{1} \Phi_{g 1}^{\mathrm{MO}}=\left(\chi_{\ell} \chi_{\ell}+\chi_{r} \chi_{r}\right)+\left(\chi_{\ell} \chi_{r}+\chi_{r} \chi_{\ell}\right) \\
&{ }^{1} \Phi_{g 2}^{\mathrm{MO}}=\left(\chi_{\ell} \chi_{\ell}+\chi_{r} \chi_{r}\right)-\left(\chi_{\ell} \chi_{r}+\chi_{r} \chi_{\ell}\right) \\
&{ }^{3} \Phi_{u}^{\mathrm{MO}}=\left(\chi_{\ell} \chi_{r}-\chi_{r} \chi_{\ell}\right) \\
&{ }^{1} \Phi_{u}^{\mathrm{MO}}=\left(\chi_{\ell} \chi_{r}-\chi_{r} \chi_{\ell}\right) .
\end{aligned}
$$

Thus in the molecular orbital description, ${ }^{3} \Phi_{u}$ is purely covalent, ${ }^{1} \Phi_{u}$ is primarily ionic, and the $\Phi_{g}$ states are mixtures.

If overlap between $\chi_{\ell}$ and $\chi_{r}$ is small (as it is in this case), then the ordering of states should be

$$
\begin{gathered}
{ }^{1} \Psi_{g 1}^{\mathrm{VB}}=\left(\chi_{\ell} \chi_{r}+\chi_{r} \chi_{\ell}\right)[\alpha \beta-\beta \alpha] \\
{ }^{3} \Psi_{u}^{\mathrm{VB}}=\left(\chi_{\ell} \chi_{r}-\chi_{r} \chi_{\ell}\right) \alpha \alpha \\
{ }^{1} \Psi_{u}^{\mathrm{VB}}=\left(\chi_{\ell} \chi_{\ell}-\chi_{r} \chi_{r}\right)[\alpha \beta-\beta \alpha] \\
{ }^{1} \Psi_{g^{2}}^{\mathrm{VB}}=\left(\chi_{\ell} \chi_{\ell}+\chi_{r} \chi_{r}\right)[\alpha \beta-\beta \alpha],
\end{gathered}
$$

with low-lying covalent states (42) and (43) and high-lying ionic states (44) and (45). Comparing wavefunctions we see that

$$
\begin{aligned}
& { }^{3} \Psi_{u}^{\mathrm{MO}}={ }^{3} \Psi_{u}^{\mathrm{VB}} \\
& { }^{\mathrm{VB}} \Psi_{u}^{\mathrm{MO}}={ }^{1} \Psi_{u}^{\mathrm{VB}},
\end{aligned}
$$

but the $g$ states are different. Substituting Eq. (33) and Eqs. (42) and (45) leads to

$$
\begin{aligned}
{ }^{1} \Psi_{g 1}^{\mathrm{VB}} & =\phi_{g}(1) \phi_{g}(2)-\lambda \phi_{u}(1) \phi_{u}(2), \\
{ }^{1} \Psi_{g 2}^{\mathrm{VB}} & =\phi_{u}(1) \phi_{u}(2)+\lambda \phi_{g}(1) \phi_{g}(2),
\end{aligned}
$$

where $\lambda \rightarrow 1$ as the overlap $S \rightarrow 0$.

The resulting spectrum of states for the VB and HF description is given in Fig. 4. Thus, although the singlet and triplet states should be nearly degenerate, $\mathrm{HF}$ necessarily leads to a triplet ground state.

The implications of these results for the Si(111) surface (unreconstructed) are as follows. ${ }^{34}$ Normal closed-shell HF calculations will lead to a band of states (corresponding to $\phi_{g}$ and $\phi_{u}$ of the two-orbital system) which are half occupied. Thus the HF results would suggest that the surface states are metallic. However, carrying out open-shell HF calculations, one would find that the ground state has each dangling-bond orbital spin-paired to form a ferromagnetic spin state (corresponding to the ${ }^{3} \Psi^{\mathrm{MO}}$ state of the two-orbital system). On the other hand, a proper treatment of the finite surface would lead to a ground state (analogous to ${ }^{1} \Psi_{g}^{\mathrm{VB}}$ ) having singlyoccupied orbitals on each surface $\mathrm{Si}$ spin-paired into an overall singlet state with low-lying excited states having these same orbitals paired to higher spins (analogous to ${ }^{3} \Psi_{u}^{\mathrm{VB}}$ ). For the perfect surface at $0 \mathrm{~K}$, these surface states would not be conducting.

Now consider distortions of the surface to form the $2 \times 1$ reconstructed surface. The closed-shell HF wavefunction $\left(\phi_{g} \phi_{g}\right.$ or $\left.\phi_{u} \phi_{u}\right)$ of the unreconstructed surface has equal amounts of ionic and covalent character. Thus, bad descriptions result because HF cannot describe a singlet pair of radical centers. If the geometry is allowed to readjust, the $\mathrm{HF}$ wavefunction will be biased toward geometries which do not lead to radical centers. This can be achieved by distorting one $\mathrm{Si}$ atom so as to stabilize a positive center (moving the Si down) and the adjacent $\mathrm{Si}$ so as to stabilize a negative center (moving the Si up),

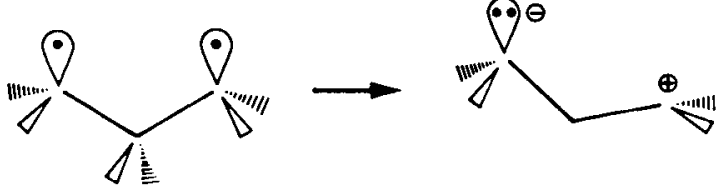

Thus the HF wavefunction is biased toward structures that stablize zwitterion character.

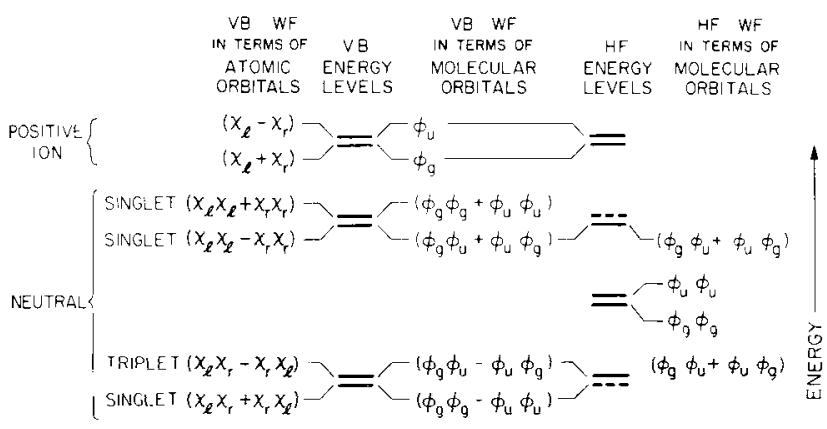

FIG. 4. Comparison of the VB and MO description for a system with separated singly-occupied orbitals. 
Recent experimental studies by Mönch ${ }^{35}$ indicate that in the $2 \times 1$ structure of $\mathrm{Si}(111)$, one $\mathrm{Si}$ moves down by $0.16 \AA$ with respect to the unreconstructed surface, while the other moves down by $0.00 \AA \hat{A}$. In comparison we have shown ${ }^{30,36}$ that a normal dangling bond $\mathrm{Si}$ relaxes down by $0.08 \AA$, the positive ion relaxes down by $0.38 \AA$, and the negative ion relaxes up by $0.17 \AA$. Thus the average displacement of the surface $\mathrm{Si}$ is just that expected of a dangling bond orbital, and the individual displacements are much less than that expected for the ionic species. However, accurate $\mathrm{HF}$ calculations for the $2 \times$ 1 structure of $\mathrm{Si}(111)$ would probably lead to distortions of approximately -0.38 and +0.17 , as expected for the ion forms.

\section{D. $\operatorname{Si}(100)$}

As an additional example, consider the reconstruction of the $\mathrm{Si}(100)$ surface. Each surface $\mathrm{Si}$ is bonded to two subsurface $\mathrm{Si}$, leading to
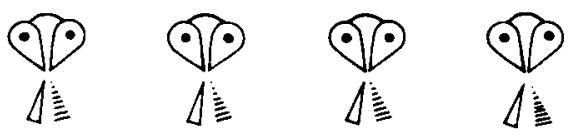

but bonding these surface atoms in pairs ${ }^{37}$ leads to
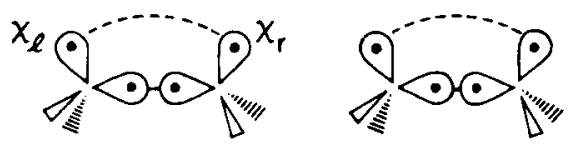

That is, one can form one strong bond between each $\mathrm{Si}$, but this leaves a radical (dangling bond) orbital on each Si that has only a small overlap with other dangling bond orbitals. The ground state of this system would be a nondegenerate (nonconducting) singlet. ${ }^{34}$

Now consider the results of a normal (closed-shell) HF calculation. The symmetrical structure of $(47)$ would lead to equal amounts of radical and ionic character and hence to a narrow surface band that is half filled (as discussed in previous reactions), that is, metallic surface states. Allowing the structure to relax with one Si of each pair moving down (stabilizing a positive center) and one Si moving up (stabilizing a negative center)

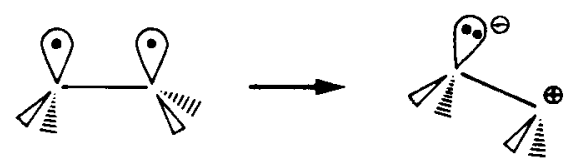

should remove the biradical character and hence a much lower energy for the $\mathrm{HF}$ wavefunction.

Indeed, approximate HF calculations on a surface model like (47) led to semimetallic surface states. ${ }^{38}$ Since experimental evidence is that the surface is not metallic, ${ }^{39}$ this result was used to argue against a pairing model such as (47) ${ }^{39}$ Calculations on a structure (48) were found to remove this metallic character, and it was suggested that the $\mathrm{Si}(100)$ surface might involve geometries such as (48).

The point in this analysis is that such results are peculiar to defects in the $\mathrm{HF}$ wavefunction and need not be relevant to the real surface.

\section{SUMMARY AND CONCLUSIONS}

The techniques of quantum chemistry have been developed to provide structure, bond energies, and reaction pathways for molecules. Those techniques based upon a single-particle approximation to the wavefunction (e.g., HF) have varying success in calculating accurately various properties. For example, $\mathrm{HF}$ is known to give rather good geometries. However, it gives rather poor values for bond energies and describes certain biradical states very poorly. To obtain more reliable values for bond energies and to obtain a valid description of the orbitals on radicals, one must employ schemes containing electron correlation effects. One such technique suitable also for studies on surfaces is GVB. The GVB method contains the dominant electron-electron correlation effects and provides orbitals suitable for a limited CI wavefunctions yielding accurate values for the bond energies, reaction surfaces, and structure.

Since the primary questions about surfaces and interfaces are often analogous to the ones quantum chemistry answers on molecular systems, we suggest that these techniques will be very useful in the field of surfaces and interfaces. The major deficiency in ab initio methods that include electron correlation effects is that it is not yet possible to study infinite systems. Consequently, it is necessary to model the surface with a finite clusters of atoms. This approach often appears particularly inappropriate to the solid state physicist who is trained to think about the importance of long-range order and delocalized states. However, processes involving reactions have long been adequately described by chemists in terms of localized models, and even on surfaces it is plausible that the chemistry should be dominated by local effects. Since many of the important questions about surfaces and interfaces involve reactions or chemistry, such approaches should provide useful information. Thus, although such approaches may be unsuccessful at predicting those features of the electronic spectrum arising from the infinite size of the system, we believe that application of these theoretical quantum chemical methods will lead to genuine insights into the phenomena at surfaces and interfaces. Even so, the theoretical challenge is to extend these methods to very large clusters or also to find some method of modeling the electronic wavefunction of the cluster with the properties of the semi-infinite system in which it should be imbedded.

\section{ACKNOWLEDGEMENT}

This work was supported in part by a grant from the National Science Foundation (No. DMR74-04965).

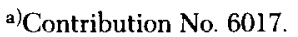

${ }^{1}$ Modern Theoretical Chemistry: Methods of Electronic Structure Theory, edited by H. F. Schaefer III (Plenum, New York, 1977), Vol. III.

${ }^{2}$ Modern Theoretical Chemistry: Applications of Electronic Structure Theory, edited by H. F. Schaefer III (Plenum, New york, 1977), Vol. IV.

${ }^{3}$ F. W. Bobrowicz and W. A. Goddard III, Ref. 1, pp. 79-128.

${ }^{4}$ A. C. Wahl and G. Das, Ref. 1, pp. 51-78.

${ }^{5}$ I. Shavitt, Ref. 1, pp. 189-276.

${ }^{6}$ J. W. Moskowitz and C. C. Snyder, Ref. 1, pp. 387-412.

${ }^{7}$ J A. Pople, Ref. 2, pp. 1-28.

${ }^{8}$ We use Hartree atomic units which are defined so that $h=1, m_{\ell}=1,|e|$
} 
$=1$. Thus the unit of energy is the hartree $=27.2116 \mathrm{eV}$, the unit of length is the bohr $=0.529177 \AA$, and the unit of velocity is $c / 137.036$. In these units, kinetic energy is $-1 / 2 \nabla^{2}$.

9(a) T. H. Dunning, Jr., J. Chem. Phys. 53, 2823 (1970); (b) T. H. Dunning, Jr., and P. J. Hay, Ref. 1, pp. 1-28.

${ }^{10}$ W. A. Lathan, L. A. Curtiss, W. J. Hehre, J. B. Lisle, and J. A. Pople, Prog. Phys. Org. Chem. 11, 175 (1974).

${ }^{11}$ Reference 2, pp. 29-108.

12W. A. Goddard III, Phys. Rev. 182, 48 (1969); P. J. Hay and W. A. Goddard III, Chem. Phys. Lett. 9, 356 (1971).

${ }^{13}$ L. B. Harding and W. A. Goddard III, J. Am. Chem. Soc. 99, 4520 (1977).

${ }^{14}$ Ignoring normalization.

${ }^{15}$ (a) W. A. Goodard III, T. H. Dunning, Jr., W. J. Hunt, and P. J. Hay, Acc. Chem. Res. 6, 368 (1973); (b) W. J. Hunt, P. J. Hay, and W. A. Goddard III, J. Chem. Phys. 57, 738 (1972).

${ }^{16}$ In both HF and GVB, several basis functions would be used (rather than just $\chi_{\ell}$ and $\chi_{r}$ ) However, the features described here would remain substantially the same.

${ }^{17}$ R. Hoffmann, J. Chem. Phys. 39, 1397 (1964).

${ }^{18}$ S. T. Pantelides, J. Vac. Sci. Technol. 16, 1349 (1979)

${ }^{19}$ R. Rein, N. Fukuda, H. Win, and G. A. Clarke, J. Chem. Phys. 45, 4743 (1966).

${ }^{20} \mathrm{~W}$. L. Bloemer and B. L. Bruner, Chem. Phys. Lett. 17, 452 (1972).

${ }^{21} \mathrm{~J}$. A. Pople and D. L. Beveridge, Approximate Molecular Orbital Theory (McGraw-Hill, New York, 1970).

${ }^{22}$ R. C. Bingham, M. J. S. Dewar, and D. H. Lo, J. Am. Chem. Soc. 97, 1285 (1975); M. J. S. Dewar and W. Thiel, J. Am. Chem. Soc. 99, 4899 (1977).
${ }^{23}$ D. J. Chadi, Phys. Rev. Lett. 41, 1062 (1978).

${ }^{24} \mathrm{~J}$. W. D. Connolly, in Modern Theoretical Chemistry: Semiempirical Methods-Techniques, edited by G. A. Segal (Plenum, New York, 1977), pp. 105-132.

${ }^{25}$ R. P. Messmer, J. Vac. Sci. Technol. 16, 1328 (1979)

${ }^{26}$ (a) J. W. P. Connolly and J. R. Sabin, J. Chem. Phys. 56, 5529 (1972); (b) U. Wahlgren, Chem. Phys. Lett. 20, 246 (1973)

${ }^{27}$ R. P. Messmer and D. R. Salahub, J. Chem. Phys., 65, 779 (1976)

${ }^{28}$ P. J. Hay and T. H. Dunning, Jr., J. Chem. Phys. 67, 2290 (1977)

${ }^{29}$ B. J. Moss and W. A. Goddard III, J. Chem. Phys. 63, 3523 (1975)

${ }^{30} \mathrm{~W}$. A. Goddard III, J. J. Barton, A. Redondo, and T. C. McGill, J. Vac. Sci. Technol. 15, 1274 (1978)

${ }^{31}$ L. B. Harding and W. A. Goddard III, J. Am. Chem. Soc. (submitted).

${ }^{32}$ P. J. Hay and W. A. Goddard III, Chem. Phys. Lett. 14, 46 (1972); P. J. Hay, and T. H. Dunning, Jr., and W. A. Goddard III, Chem. Phys. Lett. 23, 457 (1973); J. Chem. Phys. 62, 3912 (1975).

${ }^{33}{ }^{1} \Psi_{g 2}^{\mathrm{VB}}$ as written is not correct. It overlaps ${ }^{1} \Psi_{g 1}^{\mathrm{VB}}$ and hence should be orthogonalized to ${ }^{\mathrm{l}} \psi_{\mathrm{g} 1}^{\mathrm{VB}}$.

${ }^{34}$ Quantitative results are presented in A. Redondo, Ph.D. Thesis, (California Institute of Technology, 1976).

${ }^{35}$ W. Mönch, R. Feder, and P. P. Aver. J. Vac. Sci. Technol. 16, 1238 (1979).

${ }^{36}$ A. Redondo, W. A. Goddard III, T. C. McGill, and G. T. Surratt, Solid State Commun. 21, 991 (1977).

${ }^{37}$ R. E. Schlier and H. E. Farnsworth, J. Chem. Phys. 30, 917 (1959); J. D. Levine, Surf. Sci. 34, 90 (1973).

38J. P. Appelbaum, G. A. Baraff, and D. R. Hamann, Phys. Rev. Lett. 35, 729 (1975).

${ }^{39}$ F. J. Himpsel and D. E. Eastman, J. Vac. Sci. Technol. 16, 1302 (1979). 